\title{
LE RATIONNEMENT COLLECTIF DES PORCS SUIVANT « LE SEXE » ET LES CONDITIONS D'ENVIRONNEMENT
}

\author{
B. DESMOULIN \\ avec la collaboration technique de J. Rettagliati et B. Dabiel \\ Station de Recherches sur l'Élevage des Porcs, \\ Centre national de Recherches zootechniques, I. N. R. A., \\ 78 -Jouy-en-Josas
}

\section{RÉSUMÉ}

Cette étude concerne le rationnement collectif des porcs mâles castrés ou femelles dans des conditions saisonnières variables. Les plans d'alimentation antérieurement établis pour les mâles castrés (Plan I) et les femelles (Plan II) soumis à une alimentation individuelle (Desmoulin, I969) sont testés sur des groupes de 6 animaux dont la croissance débute soit en Hiver, soit en Été. Par ailleurs sont étudiés les effets d'une restriction collective de $25 \mathrm{p}$. I oo du niveau d'alimentation en distribuant les mêmes quantités de nourriture à des groupes de 8 animaux. Les résultats sont les suivants.

Lors d'une croissance débutée en période froide (Hiver) les consommations moyennes journalières d'aliment correspondent aux plans d'alimentation adoptés pour les groupes de 6 porcs. Par contre, lors d'une croissance débutée en période chaude (Été), est enregistrée une sous-consommation spontanée de $27 \mathrm{p}$. Ioo chez les castrats et de I7 p. roo chez les femelles. L'influence de la saison sur l'appétit des porcs masque alors les différences de comportement alimentaire précédemment attribuées au " sexe ". Ainsi, les performances de croissance peuvent être semblables à Haut niveau d'ingestion ( $2,5 \mathrm{~kg}$ d'aliment/jour) au cours d'une répétition Hiver-Été ou à Bas niveau d'ingestion ( $I, 9 \mathrm{~kg}$ d'aliment/jour) au cours d'une répétition Été-Hiver consécutive à la précédente.

Les restrictions imposées aux animaux en groupe de 8 sont rapportées à l'ingestion variable de nourriture enregistrée selon la saison dans les groupes Témoin de 6 porcs. On peut distinguer, par ailleurs, les niveaux de rationnement :

a) Lorsque la restriction est modérée, la croissance des mâles castrés est sévèrement réduite de I $50 \mathrm{~g} /$ jour entre 25 et Ioo $\mathrm{kg}$ de poids vif, sans que l'état d'engraissement soit influencé. La croissance des femelles est alors limitée de $85 \mathrm{~g} / \mathrm{jour}$, mais l'amélioration de la qualité des carcasses se traduit par une augmentation de $22 \mathrm{p}$. Ioo du rapport Longe/Bardière.

b) Les restrictions très ou trop sévères (notamment pour les femelles) entraînent des croissances médiocres. On note alors une amélioration de $7 \mathrm{p}$. I oo du rapport Longe/Bardière qui est équivalente chez les castrats et les femelles mais qui n'est plus valorisée par le classement des femelles.

En définitive, le rationnement collectif des porcs dans des conditions variables d'environnement permet de mieux justifier la séparation des porcs mâles castrés et des femelles. Les modalités pratiques des rationnements saisonniers restent encore à préciser. 


\section{INTRODUCTION}

Le Porc en croissance est logé en collectivité dans des bâtiments plus difficiles à climatiser en saison chaude qu'en saison froide. Aux multiples contraintes d'environnement, s'ajoutent celles du rationnement qui est imposé pour limiter l'engraissement. Les performances des pores en collectivité étant ainsi très hétérogènes (BRAUDE, I967), la transposition des résultats obtenus par ailleurs en loge individuelle est quelquefois contestée. Il est donc nécessaire de rechercher dans les méthodes d'alimentation ou les méthodes d'élevage en groupe les principales causes de cette variabilité accrue des performances.

En alimentation libérale, les régimes granulés sont consommés en repas plus longs et moins fréquents que les régimes présentés en farine sèche ou humidifiée dans l'auge (KIRMse et LANGE, I968; Faliu et Griess, I969 ; F́̂́VRIER, I970). Lors d'une distribution rationnée collectivement, le mode de présentation du régime devient ainsi un facteur de la compétition à l'auge. Par ailleurs, les porcs mâles castrés doivent être restreints plus sévèrement que les femelles (DESMOULIN, I968-I969; DESMOULIN et Bourdon, I97I). Lors du regroupement des porcs de "sexes" différents, à des âges et poids équivalents, la compétition entre les animaux d'une même loge se traduit par des niveaux de restriction très différents. Toute augmentation de la variabilité des performances reste finalement peu expliquée.

L'expérience suivante concerne le rationnement de groupes constitués soit de 6 porcs, soit de 8 porcs de même "sexe ". Le régime est granulé. Les niveaux de distribution proposés respectivement aux mâles castrés et aux femelles résultent d'enregistrements effectués antérieurement en loge individuelle. Deux répétitions saisonnières successives sont effectuées pour préciser quelques modalités d'application pratiques du rationnement collectif au cours de la croissance.

\section{MODALITÉS EXPÉRIMENTALES}

\section{I. - Habitat et environnement des animaux}

Dans une porcherie dite " d'engraissement "sur béton avec caillebotis, le chauffage ambiant et la ventilation dynamique par extraction n'ont pas permis de tamponner toutes les influences saisonnières. En période froicle (Hiver) la température est maintenue constante ( $5^{-1} 6^{\circ}$ ) avec une hygrométrie relative de l'air voisine de 70 à 80 p. Ioo. Par contre, en période chaude (Été) nous enregistrons des écarts journaliers tant de température, I 5 à $25^{\circ}$, que d'hygrométrie qui varie de 50 à 80 p. I

Les loges collectives $(2,5 \times 3,0 \mathrm{~m})$ sont adaptées à une charge optimale de 7 porcs à $100 \mathrm{~kg}$, avec une longueur d'auge de $33 \mathrm{~cm}$ par animal. Lorsque les animaux sont logés en groupes de 6 ou de 8 porcs, la longueur d'auge disponible est accrue ou réduite de $5 \mathrm{~cm}$ par porc. A un poids initial compris entre 23 et $27 \mathrm{~kg}$, les groupes sont constitués de porcs de même " sexe ", mâles castrés ou femelles. Différents niveaux d'alimentation sont testés suivant le dispositif expérimental ciaprès.

\section{2. - Aliments et niveaux de distribution}

Un régime unique, dont la consommation a été précédemment enregistrée pour les mâles castrés ou pour les femelles en logesindividuclles (Desmoulin, 1968) est utilisédurant toute la croissance de 25 à roo $\mathrm{kg}$ de poids vif. Sa composition centésimale est la suivante forge: 77 ; soja $44:$ ro; 
farine de poisson Norvège : 5 ; son de blé : 5 ; mélange minéral et vitaminique : 3 ). II renferme I6 p. Ioo de protéines brutes équilibrées pour la croissance et 3 ooo kcal d'énergie digestible par $\mathrm{kg}$ d'aliment frais. La teneur en matière sèche est de $86 \mathrm{p}$. roo. Les quantités d'aliment sont proposées suivant les normes du tableau I.

\section{TABLEAU I}

Échelles de distribution suivant le poids et le "sexe"

\begin{tabular}{|c|c|c|}
\hline "Sexe" & Mâles castrés & Femelles \\
\hline Niveaux & Échelle I & Échelle II \\
\hline $\begin{array}{l}\text { Tranche de poids } \\
\qquad(\mathrm{kg})\end{array}$ & $\begin{array}{l}\text { Quantité par porc } \\
(\mathrm{g} / \mathrm{j})\end{array}$ & $\begin{array}{l}\text { Quantité par porc } \\
(\mathrm{g} / \mathrm{j})\end{array}$ \\
\hline $20-2 \%$ & $1: 00$ & 1200 \\
\hline 28 & $1<00$ & 1370 \\
\hline 32 & 1600 & $15: 0$ \\
\hline 36 & 1800 & 1710 \\
\hline 40 & 2000 & 1880 \\
\hline $40-44$ & 2150 & 2000 \\
\hline 48 & 2300 & 2120 \\
\hline 52 & 2450 & 2240 \\
\hline 56 & 2600 & 2360 \\
\hline 60 & 2750 & 2480 \\
\hline $60-64$ & 2870 & 2570 \\
\hline 68 & 2990 & 2660 \\
\hline 72 & 3110 & 2750 \\
\hline 76 & 3230 & $28: 0$ \\
\hline 80 & 3350 & 2930 \\
\hline $80-84$ & 3440 & 2990 \\
\hline et $\mathrm{ss}$ & 一 & et ss \\
\hline
\end{tabular}

En loge collective, les plans d'alimentation I et II de type libéral sont appliqués aux groupes de 6 animaux. Les groupes de 8 animaux reçoivent collectivement la même quantité d'aliment selon le dispositif expérimental. La quantité présentée en granulés est distribuée en 2 repas par jour.

L'échelle de distribution I, libérale pour les mâles castrés, excède đe I 3 p. Ioo en fin decroissance le niveau de l'échelle de distribution II, libérale pour les femelles. Le dispositif expérimental permet de tester la validité de ces plans de rationnement en loges collectives de 6 animaux. Par ailleurs, les mêmes quantités d'aliments sont distribuées en loges collectives de 8 animaux : chaque unité de 8 porcs est alors soumise à une restriction alimentaire de $25 \mathrm{p}$. Ioo par rapport à l'unité correspondente de 6 porcs de même " sexe".

Dispositif Expérimental

\begin{tabular}{|c|c|c|c|c|c|c|c|c|}
\hline Quantités proposées & \multicolumn{4}{|c|}{ Échelle I } & \multicolumn{4}{|c|}{ Échelle II } \\
\hline "Sexe" & \multicolumn{2}{|c|}{ Mâles castrés } & \multicolumn{2}{|c|}{ Femelles } & \multicolumn{2}{|c|}{ Mâles castrés } & \multicolumn{2}{|c|}{ Femelles } \\
\hline Effectif/loge & 6 & 8 & 6 & 8 & 6 & 8 & 6 & 8 \\
\hline Niveaux relatifs & 100 & 75 & 100 & 75 & 87 & 65 & 87 & 65 \\
\hline Niveaux relatis | échelle II ...... & 113 & 85 & 113 & 85 & 100 & 75 & 100 & 75 \\
\hline
\end{tabular}


Deux répétitions, constituées chacune de 56 animaux, sont effectuées successivement de janvier à juillet au cours d'une période de croissance Hiver-Été (ou HE) puis de juillet à janvier au cours d'une période de croissance Été-Hiver (ou EH).

\section{3. - Mesures effectuées - Interprétation des résultats}

La quantité totale d'aliment consommée par loge permet, après enregistrement des refus, le calcul de la consommation moyenne par porc pendant la période de croissance considérée. De même, l'efficacité alimentaire moyenne est obtenue entre 25 et $100 \pm 2 \mathrm{~kg}$ de poids vif.

La vitesse de croissance et les caractéristiques corporelles font, par contre, l'objet de résultats individuels. Nous calculons la variabilité intragroupe $(\mathrm{s} \bar{x}=\mathrm{s} / \sqrt{\bar{n}})$ et les coefficients de variations des critères étudiés ( $\mathrm{CV}=s \sqrt{x}$. Ioo). Après l'abattage, la moyenne des mesures d'épaisseur de lard au rein et au dos constitue un premier critère d'adiposité. La variation du poids brut des fractions après la découpe des carcasses est ensuite étudiée. L'état d'engraissement de la I/2 carcasse est enfin estimé après la séparation de la longe et de la bardière, en utilisant ces deux composantes les plus explicatives du poids total des masses maigres et des masses grasses (DesmouliN, 1969; Desmoulin et Bourdon, I97 I I et II).

Pour l'ensemble du dispositif factoriel (échelles de distribution $\times$ sexes - restriction collective) les résultats sont analysés en tenant compte de l'effet répétition ou effet saisonnier.

\section{RÉSULTATS}

\section{I. - Le comportement alimentaire et l'environnement bioclimatique}

Les consommations moyennes journalières entre 25 et $100 \mathrm{~kg}$ de poids vif sont présentées dans le tableau I bis. L'effet de la répétition saisonnière sur 1'appétitest obtenu dans les groupes de 6 porcs non restreints.

\section{TABLEAU I bis}

Consommations moyennes journalières réelles (kg/jour) durant la croissance (25-10o $\mathrm{kg}$ ) suivant les niveaux de distribution, le sexe et la répétition saisonnière.

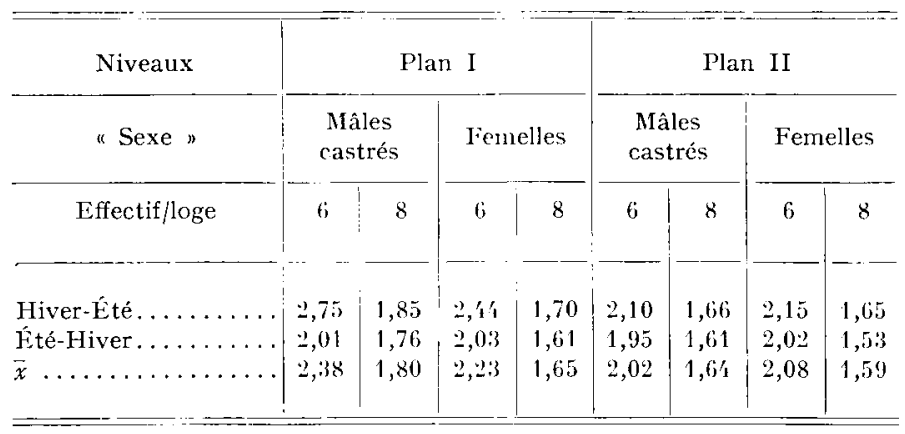

- Au cours de la répétition Hiver-Été (HE), le niveau de 1'échelle d'alimentation I libérale pour les castrats est excessif de I 2 p. Ioo pour les femelles; ces dernières sont sensiblement restreintes avec l'échelle d'alimentation II.

- Au cours de la répétition Été-Hiver (EH), la sous-consommation volontaire de nourriture est de $27 \mathrm{p}$. roo chez les castrats par rapport aux 'Témoins précédents $(2,75)$ et de $\mathrm{I} 7 \mathrm{p}$. Ioo chez les femelles par rapport aux 'Témoins de la répétition précédente $(2,44)$. Le niveau de l'échelle d'alimentation II est alors excessif pout les femell es 
Le niveau des restrictions dans les loges correspondantes de 8 porcs est donc très variable en raison de l'influence de l'environnement sur l'appétit des porcs en groupes de 6 .

\section{2. - Le niveau de consommation et la vitesse de croissance}

a) Le tableau 2 rapporte les résultats de croissance suivant les échelles d'alimentation et 1'effectif des porcs par loge. Quelle que soit la répétition saisonnière, une différenciation s'établit suivant le « sexe ».

\section{TABLEAU 2}

Vitesses de croissance $(\mathrm{g} / \mathrm{j})$ suivant la répétition saisonnière

\begin{tabular}{|c|c|c|c|c|c|c|c|c|}
\hline Niveaux & \multicolumn{4}{|c|}{ Plan I } & \multicolumn{4}{|c|}{ Plan II } \\
\hline "Sexe" & Mâles & castrés & Fer & lles & Mâles & astrés & $\mathrm{Fer}$ & tles \\
\hline Effectif/loge & 6 & 8 & 6 & 8 & 6 & 8 & 6 & 8 \\
\hline 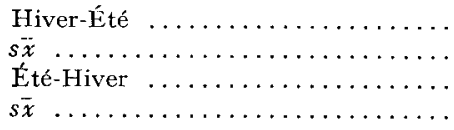 & $\begin{array}{l}702_{a} \\
43 \\
555_{b} \\
38\end{array}$ & $\begin{array}{c}503_{b c} \\
9 \\
447_{c} \\
23\end{array}$ & $\begin{array}{l}605_{b} \\
71 \\
500_{b c} \\
20\end{array}$ & $\begin{array}{l}476_{c} \\
16 \\
455_{c} \\
21\end{array}$ & $\begin{array}{c}467_{c} \\
18 \\
517_{b} \\
24_{4}\end{array}$ & $\begin{array}{c}434_{c} \\
11 \\
435_{c} \\
17\end{array}$ & $\begin{array}{l}550_{c} \\
52 \\
526 b \\
26\end{array}$ & $\begin{array}{c}471_{c} \\
22 \\
440_{c} \\
7\end{array}$ \\
\hline $\begin{array}{l}\bar{x}, \ldots \ldots \ldots \ldots \ldots \ldots \ldots \ldots \ldots \ldots \ldots \\
\mathrm{CV}(\%) \ldots \ldots \ldots \ldots \ldots \ldots \ldots \ldots \ldots \ldots\end{array}$ & $\begin{array}{c}621_{b} \\
19\end{array}$ & $\begin{array}{c}475_{c} \\
12\end{array}$ & $\begin{array}{c}550_{b c} \\
25\end{array}$ & $\begin{array}{c}465 c \\
10\end{array}$ & $\begin{array}{l}492 b c \\
11\end{array}$ & $\begin{array}{c}435_{c} \\
9\end{array}$ & $\begin{array}{c}538_{b c} \\
19\end{array}$ & $\begin{array}{c}455_{c} \\
9\end{array}$ \\
\hline
\end{tabular}

Les valeurs portant la même lettre en indice ne diffèrent pas au seuil $\mathbf{P}<0,05$.

- Dans les groupes de 6 porcs, la croissance des castrats est supérieure de 50 à roo g/ jour à celle des femelles lorsque l'échelle d'alimentation I est libérale. Par contre, la croissance des castrats est inférieure en moyenne de $45 \mathrm{~g} /$ jour à celle des femelles avec 1'échelle d'alimentation II moins libérale.

- Les résultats obtenus dans les groupes de 8 porcs confirment cette différence d'adaptation aux restrictions alimentaires : la croissance des castrats est réduite de I Io à $200 \mathrm{~g} /$ jour alors qu'elle est réduite de 45 à I $30 \mathrm{~g} / \mathrm{jour}$ chez les femelles.

Ainsi les restrictions alimentaires obtenues " par compétition " en augmentant la densité d'occupation des loges collectives sont très sévères. Par ailleurs, le coefficient de variation des performances de croissance est plus faible dans les groupes de 8 porcs ( 9 à I 2 p. IOo) que dans les groupes de 6 porcs (II à 25 p. IOO). Ce résultat traduit une grande homogénéité des croissances lors des restrictions sévères.

b) Sur la figure $\mathrm{I}$, la réponse de croissance aux variations des niveaux d'alimentation est présentée suivant le " sexe " et les conditions saisonnières. Quelles que soient ces dernières, la pente de ces droites de réponse indique une baisse de la vitesse de croissance plus importante chez les castrats que chez les femelles lors d'une même réduction du niveau d'alimentation. Par ailleurs, les températures saisonnières excessives en début de croissance ont été très préjudiciables au niveau global des performances 
obtenues atu cours de la répétition Été-Hiver (ou $\mathrm{EH}$ ). Ces graphiques permettent les conclusions suivantes : les vitesses de croissance des castrats et des femelles sont semblables à Haut-niveau d'ingestion $(2,55 \mathrm{~kg} / \mathrm{j}$ our $)$ au cours d'une séquence de type HiverÉté d'environnement favorable à la croissance. Par contre, l'égalisation est obtenue entre " sexes " à Bas-niveau d'ingestion ( $I, 87 \mathrm{~kg} / \mathrm{jour})$ dans un environnement de type Été-Hiver défavorable à la croissance.
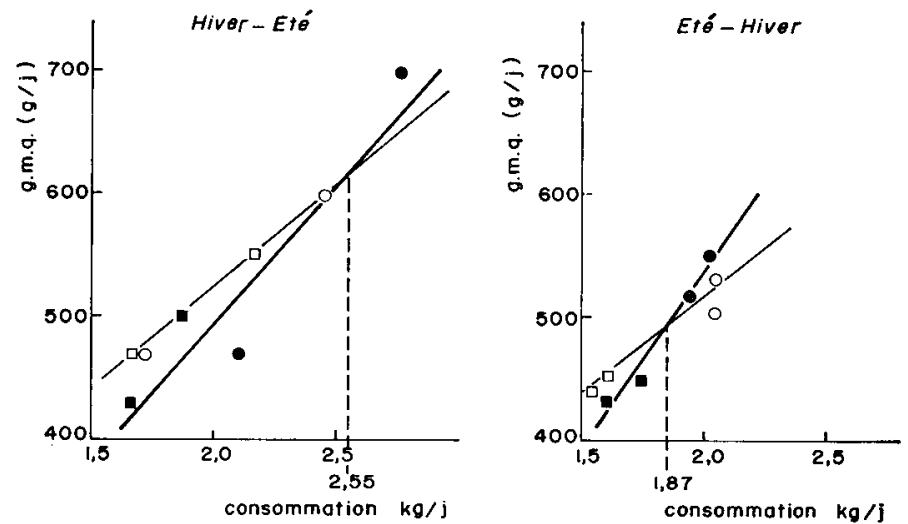

FIG. I. - Influences saisonnières sur la vitesse de croissance en alimentation collective des groupes de 6 ou 8 porcs

Mâles castrés

Femelles

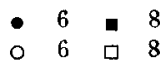

\section{3. - L'efficacité alimentaire globale et la sévérité du rationnement}

Dans le tableau 3, les consommations totales d'aliment pour un même gain de poids sont présentées suivant les niveaux de distribution et le sexe.

TABLEAU 3

Consommation totale d'aliment par porc $(\mathrm{kg})$ suivant le sexe et la succession des saisons

\begin{tabular}{|c|c|c|c|c|c|c|c|c|}
\hline Niveaux & \multicolumn{4}{|c|}{ Plan I } & \multicolumn{4}{|c|}{ Plan II } \\
\hline "Sexe " & \multicolumn{2}{|c|}{$\begin{array}{c}\text { Mâles } \\
\text { castrés }\end{array}$} & \multicolumn{2}{|c|}{ Femelles } & \multicolumn{2}{|c|}{$\begin{array}{c}\text { Mâles } \\
\text { castrés }\end{array}$} & \multicolumn{2}{|c|}{ Femelles } \\
\hline Effectif/loge & 6 & 8. & 6 & 8 & 6 & 8 & 6 & 8 \\
\hline Hiver-Été . . . . . . . . . & 295 & 276 & 303 & 267 & 337 & 287 & 292 & 262 \\
\hline Été-Hiver.......... & 271 & 296 & 304 & 265 & 281 & 278 & 287 & 261 \\
\hline $\bar{x} \ldots \ldots \ldots \ldots \ldots$ & 283 & 286 & 303 & 266 & 310 & 282 & 290 & 261 \\
\hline
\end{tabular}

- En alimentation très libérale $(\mathrm{I})$, la dépense totale de consommation dans les groupes de 6 porcs est comparable chez les castrats et les femelles dont l'appétit s'extériorise pleinement. Entre 25 et Ioo $\mathrm{kg}$ de poids vif, cette dépense est voisine de $300 \mathrm{~kg}$ d'aliment frais. 
- La diminution du niveau de distribution (II) et surtout les restrictions alimentaires consécutives à l'augmentation des effectifs abaissent cette dépense totale de 29 à $37 \mathrm{~kg}$ d'aliment chez les femelles. L'influence saisonnière ne peut toutefois être dissociée de celle propre au niveau de la restriction. Ainsi, les tendances divergentes obtenues chez les mâles castrés suivant la saison traduisent le résultat suivant : l'efficacité alimentaire, peu améliorée par une restriction modérée, est réduite lors d'une restriction sévère. Entre 25 et Ioo $\mathrm{kg}$ de poids vif, le rationnement des castrats est obtenu avec $284 \mathrm{~kg}$ d'aliment $(\mathrm{IC} \# 3,70)$ alors qu'il est obtenu avec $263 \mathrm{~kg}$ d'aliment (IC \# 3,40) chez les femelles. En loge collective, les femelles maintiennent, par ailleurs, ces dépenses totales d'aliment plus constantes dans des conditions saisonnières variables.

\section{4. - La composition des carcasses: infuences du sexe et de l'environement}

Pour l'ensemble des carcasses des porcs de race Large-White, l'épaisseur moyenne du lard dorsal est de 28 , I mm chez les castrats contre $24,6 \mathrm{~mm}$ chez les femelles. Les résultats du tableau 4 mettent en évidence 1'influence saisonnière sur ce premier critère d'adiposité. La période de finition en Hiver est favorable à une réduction du dépôt gras sous-cutané, lorsque la sous-consommation volontaire en début de croissance

TABLEAU 4

Épaisseur moyenne du lard dorsal (mm) au poids d'abattage : $100 \pm 2 \mathrm{~kg}$

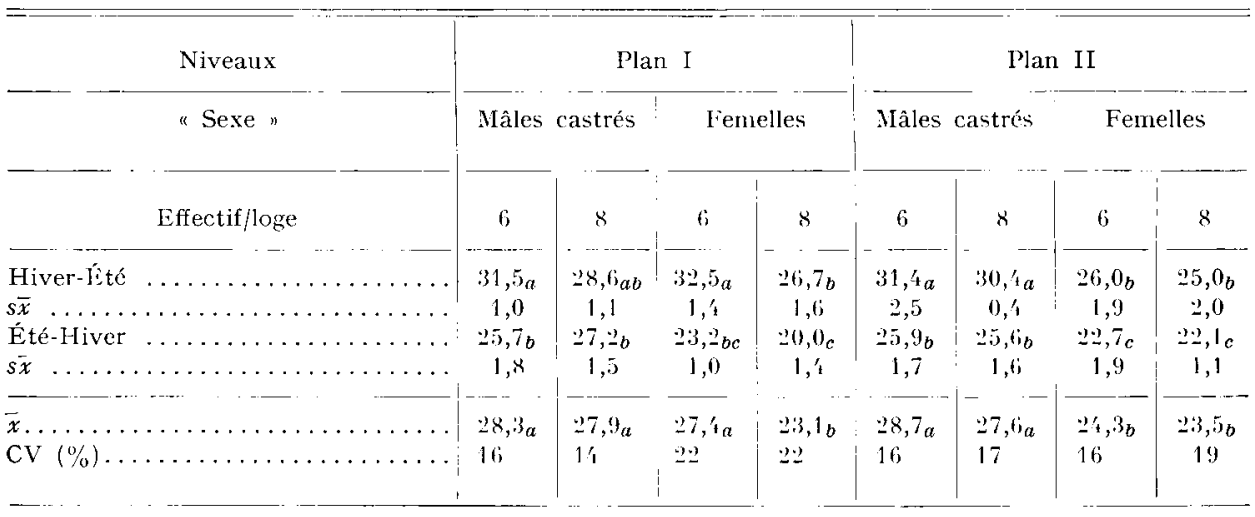

Les valeurs portant la même lettre ne different pas au seuil $\mathrm{P}^{\mathrm{S}}<0,05$.

d'Été est très, sinon trop, efficace en particulier pour les femelles restreintes. Ces dernières présentent 20 à $22 \mathrm{~mm}$ d'épaisseur du lard dorsal. Inversement, la finition en Été met en évidence le risque d'une alimentation très libérale des femelles lorsque celles-ci ont pleinement extériorisé leur appétit en début de croissance d'Hiver. L'épaisseur moyenne du lard dorsal de $32,5 \mathrm{~mm}$ obtenue dans le groupe des 6 pores femelles au cours de la croissance Hiver-H́té traduit ce risque important. La restriction alimentaire précoce évite ce risque. 
Les poids bruts des morceaux après la découpe sont présentés dans le tableau 5 . Le poids du jambon est bien un critère peu objectif des variations de composition corporelle. Les poids de L,onge et de Bardière subissent des variations plus cohérentes, les coefficients de variation étant respectivement de 5 à $6 \mathrm{p}$. Ioo pour la masse maigre et de $\mathrm{I} 5$ à $\mathrm{I} 6 \mathrm{p}$. Ioo pour la masse grasse de l'ensemble Longe + Bardière. Enn utilisant le rapport entre ces poids bruts comme index qualitatif, les résultats du tableau 6 précisent l'importance des facteurs de variations de la composition des carcasses. Les résultats sont différents suivant le « sexe » et le niveau alimentaire.

TABI,EAU 5

Poids moyen $(\mathrm{kg})$ des morceaux séparés après la découpe des demi-carcasses sans tête

\begin{tabular}{|c|c|c|c|c|c|c|c|c|c|}
\hline \multirow{3}{*}{\multicolumn{2}{|c|}{$\frac{\text { Niveaux }}{\text { "Sexe" }}$}} & \multicolumn{4}{|c|}{ Plan I } & \multicolumn{4}{|c|}{ Plan II } \\
\hline & & \multicolumn{2}{|c|}{ Mâles castrés } & \multicolumn{2}{|c|}{ Femelles } & \multicolumn{2}{|c|}{ Mâles castrés } & \multicolumn{2}{|c|}{ Femelles } \\
\hline & & 6 & 8 & 6 & 8 & 6 & 8 & 6 & 8 \\
\hline 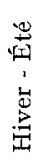 & $\begin{array}{l}1 / 2 \text { carcasse } \ldots \ldots \ldots \\
\text { Jambon } \ldots \ldots \ldots \ldots \\
\text { Longe } \ldots \ldots \ldots \ldots \\
\text { Bardière } \ldots \ldots \ldots \ldots \\
\text { Panne } \ldots \ldots \ldots \ldots\end{array}$ & $\begin{array}{r}37,10 \\
7,79_{a} \\
10,90_{a} \\
6,13_{a} \\
1,21_{a}\end{array}$ & $\begin{array}{c}36,70 \\
7,91_{a} \\
10,70_{a b} \\
5,85 b \\
1,11_{a b}\end{array}$ & $\begin{array}{r}37,20 \\
7,73_{a} \\
10,45_{b} \\
6,52_{a} \\
1,28_{a}\end{array}$ & $\begin{array}{r}36,00 \\
8,06_{a} \\
10,95_{a} \\
5,50_{b} \\
0,97_{b}\end{array}$ & $\begin{array}{r}37,20 \\
7,91_{a} \\
10,4_{a} \\
6,31_{a} \\
1,26_{a}\end{array}$ & $\begin{array}{c}36,50 \\
7,32_{b} \\
10,73_{a b} \\
6,34_{a} \\
1,30_{a}\end{array}$ & $\begin{array}{r}36,50 \\
7,72_{a} \\
10,92_{a} \\
5,50_{b} \\
1,01_{b}\end{array}$ & $\begin{array}{r}36,10 \\
7,91_{a} \\
11,07_{a} \\
5,28_{b} \\
0,96_{b}\end{array}$ \\
\hline 离 & $\begin{array}{l}1 / 2 \text { carcasse } \ldots \ldots \ldots \\
\text { Jambon } \ldots \ldots \ldots \ldots \\
\text { Longe } \ldots \ldots \ldots \ldots \\
\text { Bardière } \ldots \ldots \ldots \ldots \\
\text { Panne } \ldots \ldots \ldots \ldots\end{array}$ & $\begin{array}{r}35,90 \\
7,76_{a} \\
10,87_{a} \\
5,25_{b} \\
0,87_{b}\end{array}$ & $\begin{array}{r}36,55 \\
7,76_{a} \\
10,40_{b} \\
5,77_{b} \\
1,22_{a}\end{array}$ & $\begin{array}{r}36,10 \\
7,90_{a} \\
10,52_{b} \\
5,38_{b} \\
1,03_{a}\end{array}$ & $\begin{array}{r}35,10 \\
7,81_{a} \\
10,84_{a} \\
4,57_{c} \\
0,83_{b}\end{array}$ & $\begin{array}{l}35,30 \\
7,56_{a b} \\
10,37_{b} \\
5,4_{b} \\
1,00_{a b}\end{array}$ & $\begin{array}{c}35,70 \\
7,55_{a b} \\
10,76_{a b} \\
5,18_{b} \\
1,0^{\prime} 4_{a}\end{array}$ & $\begin{array}{r}36,50 \\
8,00 a \\
10,96_{a} \\
5,06_{b} \\
0,93_{b}\end{array}$ & $\begin{array}{r}35,10 \\
7,86_{a} \\
10,92_{a} \\
4,66_{c} \\
0,85_{b}\end{array}$ \\
\hline
\end{tabular}

Les valeurs portant la même lettre ne diffèrent pas au seuil $\mathrm{P}<0,05$.

TABLEAU 6

Relation poids longe/poids de bardiève

\begin{tabular}{|c|c|c|c|c|c|c|c|c|}
\hline Niveaux & \multicolumn{4}{|c|}{ Plan I } & \multicolumn{4}{|c|}{ Plan II } \\
\hline "Sexe" & \multicolumn{2}{|c|}{$\begin{array}{l}\text { Mâles } \\
\text { castrés }\end{array}$} & \multicolumn{2}{|c|}{ Iiemelles } & \multicolumn{2}{|c|}{$\begin{array}{c}\text { Mâles } \\
\text { castrés }\end{array}$} & \multicolumn{2}{|c|}{ Femelles } \\
\hline Effectif/loge & 6 & 8 & 6 & 8 & 6 & 8 & 6 & 8 \\
\hline Hiver-f́té. & 1,69 & $1,8: 3$ & 1,60 & 1,99 & 1,65 & 1,69 & 1,98 & 2,05 \\
\hline Été-Hiver... & 2,07 & 1,80 & 1,95 & 2,37 & 1,91 & 2,08 & 2,16 & $2,3^{\prime}$ \\
\hline $\bar{x} \ldots \ldots$ & 1,88 & 1,81 & 1,77 & 2,18 & 1,78 & 1,88 & 2,07 & 2,21 \\
\hline
\end{tabular}

- Lors d'une alimentation très libérale (plan I), le rapport Longe/Bardière chez les femelles est supérieur de 7 p. Ioo à celui des castrats. En alimentation rationnée (plan II) les femelles présentent un index qualitatif supérieur de 17 p. Iooà celui des castrats. 
- Les restrictions obtenues par augmentation des effectifs améliorent de 22 p. Ioo la qualité de carcasse des femelles, alors qu'elles ont des influences nulles ou contradictoires chez les castrats. Lors des restrictions les plus sévères, l'augmentation ultérieure du rapport Longe/Bardière devient moindre $(+6$ à +7 p. Ioo) mais équivalente chez les castrats et les femelles. Les niveaux de ces améliorations sont alors nettement distincts suivant le " sexe ».

\section{DISCUSSION ET CONCLUSION}

Les résultats de cette expérience posent deux questions préliminaires à l'établissement du rationnement collectif des porcs suivant les conditions d'environnement et le mode de constitution des groupes d'animaux.

\section{Infuence de l'environnement sur les performances de croissance}

Les échelles de distribution pré-établies en porcherie disposant de loges pour une alimentation individuelle ne sont pas adaptées à toutes les conditions d'une alimentation collective. Les différences sont liées conjointement au type de bâtiment et d'environnement des animaux. Toutefois la maîtrise du confort thermique de l'animal est particulièrement importante.

a) Lors d'une croissance débutée en période froide (Hiver) les porcs en collectivité présentent des performances semblables à celles obtenues en loge individuelle sans restriction alimentaire (DEsmoulin, I969). Par contre, 1'influence d'une période chaude (Été) en début de croissance se traduit par une diminution moyenne de 20 p. Ioo de la consommation spontanée par rapport aux quantités proposées aux animaux. Selon Seymour et al. (I964), Holme et Cofy (I967), Hal et al. (I968) le confort thermique est voisin de $I 5^{-I} 6^{\circ} \mathrm{C}$ chez le porc en croissance ; ces valeurs sont celles enregistrées dans la porcherie expérimentale en Hiver. Par contre, en accord avec LEROY (I953), les jeunes porcs subissent très fortement l'effet dépressif des températures excessives dans la porcherie $\left(25^{\circ} \mathrm{C}\right)$ en Été. Selon Grosse et PFEIFFER, I963, les températures trop basses ( $\mathrm{IO}^{\circ} \mathrm{C}$ ) en Hiver sont défavorables. Toutefois JENSEN et al. (I969) mettent en évidence l'influence stimulante du froid sur le niveau d'ingestion pour maintenir la vitesse de croissance. Le défaut de ventilation des bâtiments soumis à des températures excessives est donc très préjudiciable. Les variations du degré d'hygrométrie ne constituent pas, selon ADDIs et al., (I967), un facteur essentiel du confort climatique de l'animal.

b) La surpopulation provoquée dans les loges de 8 pores en vue d'effectuer un rationnement déprime très fortement la vitesse de croissance. Plus accentuée chez les castrats que chez les femelles, la variabilité des résultats de croissance paraît liée, comme l'indiquait MADSEN (I966), au niveau de la consommation. Les variations d'appétit attribuées à l'environnement collectif (JENSEN, I967; HoL,MEs et MounT, I967) peuvent correspondre à des dépenses thermiques ajustées au maintien de la température corporelle (Moun'T, Ig68). 
Le confort thermique doit être associé à la détermination des modalités du rationnement collectif des porcs. Par ailleurs, la période qui suit les transitions après le sevrage conditionne largement les performances globales de la croissance et, plus encore, les résultats de composition corporelle.

\section{Composition des carcasses et variations du rationnement des castrats et des femelles}

Après la découpe des carcasses, l'état d'engraissement est estimé par 1'augmentation du rapport Longe/Bardière comme index qualitatif, lorsque le poids brut du jambon reste sans signification.

Outre la supériorité systématique de la qualité des carcasses des femelles sur celles des castrats, l'effet du rationnement varie suivant le " sexe ". Les restrictions établies avec le plan d'Alimentation I, le plus libéral, permettent d'accroître de 22 p. Ioo le rapport Longe/Bardière des femelles restreintes. Les restrictions plus sévères établies avec le plan d'alimentation II, le moins libéral, permettent des améliorations plus faibles qui sont alors équivalentes pour les femelles et les castrats. En accord avec les résultats obtenus en alimentation individuelle (I)ESMOULIN, rg68-I969; DESMOULIN et BOURDON, I97I) ces différences s'expliquent par une consommation " de luxe " plus élevée d'environ I5 p. Ioo chez les castrats en alimentation libérale.

Pour la production de carcasses maigres, les restrictions modérées et précoces sont justifiées chez les femelles dont l'appétit s'extériorise pleinement au cours de la phase dite de "Pré-engraissement ". Les caractéristiques des carcasses des femelles en alimentation libérale (épaisseur de lard dorsal : 32,5 mm, rapport Longe/Bardière : I,60) montrent bien ici le risque d'un rationnement non maîtrisé au débụt de croissance en période froide (Hiver).

\section{Conséquences pratiques et conchusions}

L'absence de sensibilité des résultats moyens qui concernent un nombre égal de castrats et de femelles (Sмrтн ct al., I967) est explicitée par les différences de réponses en alimentation restreinte. $\mathrm{L}_{1}$ a discordance des résultats rapportés dans les revues bibliographiques de VANschoubrokx ct al., (1967) et BRAUDE ( 1967 ,) trouve ici quelques explications : une intensité moyenne de restriction ne peut se référer qu'aux différents niveaux d'expression des aptitudes des porcs suivant le " sexe " et les conséquences de la castration. Lorsque de nombreux auteurs justifient parallèlement une majoration des normes azotées chez les femelles (Robinson, I966; WaLlace, I965 ; RÉrAT et HExRY, I970 ; LOUGxON, I970), le choix de régimes différents de ceux utilisés chez les castrats (LEWIS et HARDY, I970) conduit en alimentation collective à la séparation inévitable des porcs suivant le " sexe ".

Le seuil de réduction accepté pour la vitesse de croissance et le seuil d'amélioration obtenu lors du classement des carcasses déterminent pratiquement l'intensité optimale de restriction. I e modèle unique de rationnement doit être abandonné. Lorsque les variations du comportement alimentaire liées à l'habitat et aux conditions climatiques sont déterminantes du niveau des performances, les modalités d'application pratique des rationnements saisonniers sont justifiées mais encore peu précisées. 


\section{SUMMARY}

\section{COLIECTIVE DISTRIBUTION OF FOOD TO PIGS ACCORDING TO THE SEX AND THE ENVIRONMENTAL CONDI'TIONS}

This study deals with the collective distribution of food to castrated male pigs or females reared under various seasonal conditions. The previously established feeding schedules for castrated males (schedule I) and females (schedule II) submitted to individual feeding (DEsmoulin, I 969) were tested on groups of 6 animals each, the growth of which began either during the winter or the summer season. In addition, we studied the effects of a collective restriction (25 per cent of the feeding level) by distributing the same amount of food to groups of 8 animals. The results were as follows :

- When the growth period started during the winter-time, the mean food intake corresponded to the feeding schedules established for the groups of 6 pigs. On the other hand, when the growth began in the summer-time, the food intake was spontaneously 27 per cent lower in the castrates and 17 per cent lower in the females. The influence of the season upon the appetite of the pigs thus conceals the differences in the feeding behaviour previously imputed to " the sex ". Thus, the growth performances may be similar during a winter-summer replicate at a high ingestion level $(2.5 \mathrm{~kg}$ food/day) or at a low ingestion level ( $1.9 \mathrm{~kg}$ food/day) during a summer-winter replicate consecutive to the former.

The food restrictions imposed to the animals ( 8 per group) have been related to the different ingestion levels according to seasons in the control groups of 6 animals each. Moreover, it was noticed that.

a) when the food restriction was moderate, the growth of the castrated males was severely reduced by $150 \mathrm{~g} / \mathrm{day}$ between 25 and Ioo $\mathrm{kg}$ live weight without any effect upon the state of fattening of the animals. The growth of the females was reduced by $85 \mathrm{~g} /$ day, but the quality of the carcasses was improved by an increase of 22 per cent of the loin/backfat ratio.

b) too important food restrictions (especially in the females) result in very bad growth rates. Under these conditions, there is an improvement of 7 per cent of the loin/backfat ratio, but as the grading of the females is already optimum. the results are not valorized.

It may be concluded that the separation of the castrated male pigs from the females is absolutely justified under the conditions of collective food distribution. The food restriction patterns according to seasons have to be defined more accurately.

\section{RÉFÉRENCES BIBLIOGRAPHIQUES}

Addis P. B., Johnson M. R., Heidenreich C. J., Jones H. W., Judge M. D., ig67. Effect of humidity level in a warm growing environment on porcine carcass composition. $\ddot{J}$, anim. Sci. 26, 705-708.

Braude R., I967. The effect of changes in feeding patterns on the performance of pigs. Proc. Nutr. Soc., 26, I63-I8I.

Desmoulin B., I968. The effect of feed restriction on pig performances and variations according to, sex. Proc. 2nd World Conf. Anim. Prod. University of Maryland. r4-20 July. U.S.A., 549.

Desmoulin B., r969. Données préliminaires relatives à la détermination de l'état d'engraissement de la carcasse du Porc. Journées Rech. porcine en France, 2 I3-2I9, I. N. R. A., Paris. 20-2 I février.

Desmoulin B., Bourdon D., i97i. La séparation des porcs mâles castrés et des porcs femelles en alimentation restreinte depuis le sevrage. Journées Rech. Porcine en France, 73-9o, I. N. R. A, Paris, I8-ı 9 février.

FAliu L., Griess, I969. Le comportement alimentaire du Porc Charcutier. Journées Rech. Porcine en France, 6r-66, I. N. R. A.. Paris, 20-2 I février,

FÉvrier C., I97o. Mode de présentation de l'aliment et comportement lors du repas, chez le Porc en croissance. Journées Rech. Porcine en France Paris, r9-20 février, I6r-I66. I.N.R.A..

Grosse F., Pfeiffer H., I963. Influence of environmental temperature on rate of gain, feed consumption and energy utilization by the pig. Nutv. Abst. Rev., $\mathbf{3 8}$, i 170 . 
Hale O. M., Johnson J. C., WarRen Jr Ep., I968. Influence of season, sex and dietary energy concentration on performance and carcass characteristics of swine. J. anim. Sci., 27, 1577-I582.

Holme D. W., Coey W. E., I967. The effects of environmental temperature and method of feeding on the performance and carcass composition of bacon pigs. Anim. Prod., 9, 209-218.

Holmes C. W., Mount L. E., 1967. Heat loss from groups of growing pigs under various conditions of environmental temperature and air movement. Anim. Prod., 9, 435-452.

Jensen A. H., 1967. Developments in environment controlled facilities for swine. Feedstuffs, 39, n 37 , I8-I9.

Jensen A. H., Kulman D. E. Becker D. E., Harmon B. E., ig69. Response of growing-finishing swine to different housing environment during winter seasons. J. anim. Sci, 29, 45 I-456.

KIRMSE K., LANGE H., r968. Verhalten von Mastschweinen bei unterschiedlicher Futterkonsistenz, Tierzucht, 22, II8.

Leroy A. M., I953. Variation des besoins alimentaires des porcs en fonction de la saison. Ann. Zootech., 1, I-9.

LEwis D., HARDY B., I97o. The effect of dietary energy and protein on carcass composition in the growing pig. Ritena Conf. Majorca. Mai.

Lougnon J., I97o. Supplémentation par la lysine et la méthionine d'aliments chez le porc en croissance finition : influence du sexe et du rationnement. Journées Rech. Porcine en France, 85-90, I. N. R. A, Paris, 19-20 février.

Madsen A., 1966. Some statistical analyses of nutrition experiments with pigs. Acta A gric. Scand., 16, I80-I93.

Mount K. E., I968. The climatic Physiology of the pig. Edward Arnold. London, 270 pp.

Rérat A., HEnRy Y., 1970. Étude du besoin en acides aminés soufrés du Porc en croissance. Journées Rech. Porcine, 6I-66, I. N. R. A. Paris, 19-2o février,

Robinson D. W., I966. Sex differences in the response of pigs to dietary amino-acid supplementation with reference to growth and body composition. J. Sci. Food. A gric., 17, I-6.

Seymour E. W., Speer V.C., Hays V.W., Mangold $\quad$ D. W., Hazen T. E., I964. Effectsof dietary protein level and environmental temperature on performance and carcass quality of growing finishing swine. J. anim. Sci., 23, 375-379.

Smith J., Clawson A. J., Barrick E. R., I967. Effect of ratio of protein from corn and soybean meal in diets of varying total protein on performance, carcass desirability and diet digestibility in swine. J. anim. Sci., 26, 752-758.

Vanschoubroek F., DE Wilde R., Lampo P. H., I967. The quantitative effect of feed restriction in fattening pigs on weight gain, efficiency of feed utilization and backfat thickness. Anim. Prod., 9, 67-74.

WALlace H. D., I965. Dietary protein level, feed restriction and sex influence on the feedlot performance and carcass characteristics of finishing swine. Feedstuffs, 37, I8-I9. 